\title{
Raised intracranial pressure presenting with spontaneous periorbital bruising: two case reports
}

\author{
S Hadjikoutis, C Carroll, G T Plant
}

J Neurol Neurosurg Psychiatry 2004;75:1 192-1193. doi: 10.1136/jnnp.2003.016006

The venous drainage of the orbit is known to be via the ophthalmic and vortex veins which communicate with the cavernous sinus. We describe two patients with raised intracranial pressure presenting with periorbital bruising. In one patient dural venous sinus thrombosis was demonstrated and it is suspected that the cause of the raised intracranial pressure may have been the same in the second. We suggest that the abrupt rise of pressure in the cerebral venous system was transmitted via the cavernous sinus to the orbital venous system.

$\mathrm{T}$ he early diagnosis of raised intracranial pressure can be problematic, especially when the patient first visits an accident and emergency department and there are no abnormal physical signs. We describe two patients who presented with headache due to raised intracranial pressure associated with bilateral periorbital bruising. We suggest that this may be an external sign of raised intracranial pressure under certain circumstances. We then go on to discuss the possible mechanisms whereby an abrupt rise in intracranial pressure may give rise to periorbital bruising.

\section{CASE REPORTS}

\section{Case 1}

A 24 year old woman with a history of migraine presented with a three day history of spontaneous periorbital bruising. One week previously she had awakened during the night with a generalised headache that increased in severity over the next 3-4 hours. It was associated with nausea, vomiting, and mild photophobia, and two days later with neck stiffness. Two days after the onset of headache she was seen by her general practitioner who diagnosed migraine. After developing bilateral periorbital bruising, she sought the advice of a number of doctors, most of whom suspected that she had been assaulted. However, she eventually presented to the casualty department of Moorfields Eye Hospital, London. On examination, there was bilateral periorbital bruising, more marked on the right (fig 1), and bilateral acute papilloedema. Computed tomographic (CT) imaging of the brain demonstrated expansion of the posterior part of the superior sagittal sinus with low density centrally. There was a small area of superficial haemorrhage superiorly in the posterior part of the right frontal lobe (fig 2). The appearances were consistent with dural sinus thrombosis with a superficial haemorrhagic venous infarct. These findings were confirmed by magnetic resonance imaging (MRI) of the brain and MR venography (MRV). The cavernous sinuses were patent and the superior ophthalmic veins were of normal calibre. No lumbar puncture was carried out, as the diagnosis was not in doubt. The patient was started on intravenous heparin and then anticoagulated with

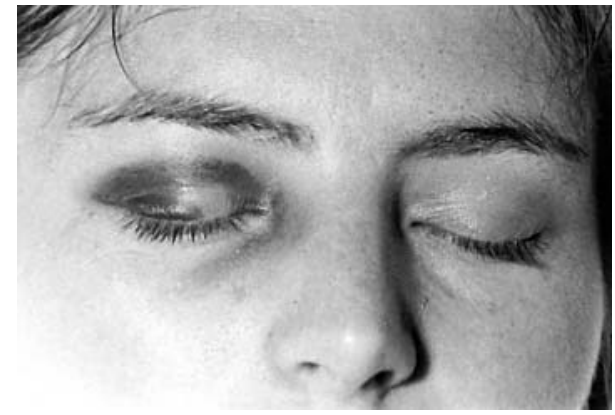

Figure 1 Case 1: periorbital bruising more marked on the right.

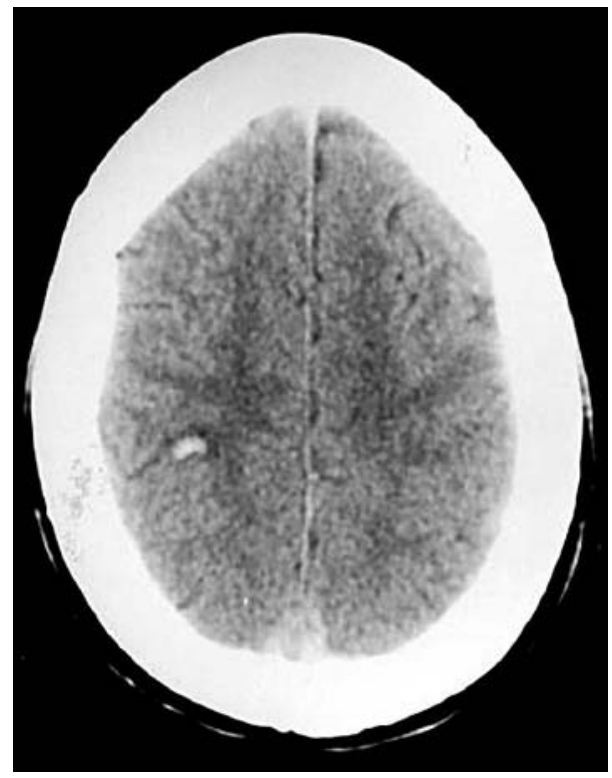

Figure 2 Case 1: Brain computed tomography scan showing expansion of the posterior part of the superior sagittal sinus with low density centrally. There is a small area of superficial haemorrhage superiorly in the posterior part of the right frontal lobe.

warfarin. The periorbital bruising resolved over the next few weeks.

\section{Case 2}

A 25 year old woman presented with a six day history of headache, neck stiffness, vomiting, and photophobia. She was 25 weeks pregnant. On examination, she had bilateral periorbital bruising, more marked on the left (fig 3), bilateral

Abbreviations: $\mathrm{CT}$, computed tomography; MRI, magnetic resonance imaging; MRV, magnetic resonance venography 


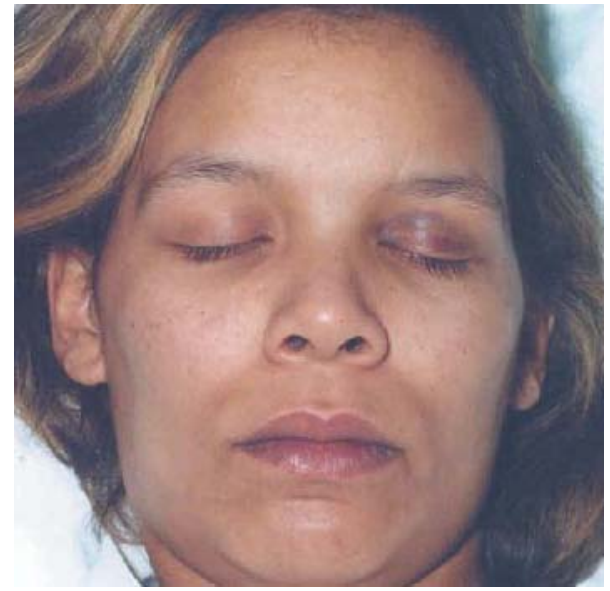

Figure 3 Case 2: periorbital bruising more marked on the left.

sixth nerve palsy and papilloedema. There was no history of trauma. MRI of the brain and MRV were normal. Lumbar puncture revealed an opening pressure of $46 \mathrm{~cm}$; constituents were normal. The headache improved following the procedure. The patient was started on acetazolamide. The periorbital bruising resolved over the next few weeks. Although dural sinus thrombosis was not confirmed, the history also suggested subacute onset of raised intracranial pressure and, in view of the fact that the patient was pregnant, dural sinus thrombosis was considered a possible cause of the clinical syndrome.

\section{DISCUSSION}

The venous drainage of the orbit is known to be via the ophthalmic and vortex veins. The superior ophthalmic vein drains the superior regions of the orbit, eyelids, and forehead. It anastomoses with the terminal twigs of the angular vein, a branch of the facial vein. The inferior ophthalmic vein (where present) drains the inferior regions of the orbit and eyelids, as well as the vortex veins from the globe. It also anastomoses with the terminal twigs of the angular vein and the pterygoid plexus. The superior and inferior ophthalmic veins leave the orbit via the superior orbital fissure to drain, either separately or by one trunk, into the cavernous sinus. We have described two patients with subacute onset of raised intracranial pressure presenting with apparently spontaneous periorbital bruising. We propose that the abrupt rise in the pressure in the cerebral venous system was transmitted via the cavernous sinus to the orbital venous system, and that this resulted in the spontaneous periorbital bruising in these patients.

Engorgement of the superior ophthalmic vein may be seen acutely in this situation but as the imaging was obtained 14 and 21 days following the onset of headache in cases 1 and 2 , respectively, any such changes would have normalised. Although we are unable to confirm that this is a hallmark specifically of dural sinus thrombosis, as the cause was not established in case 2, we suspect that this is the case. While this paper was being reviewed a photograph was published showing a case of superior sagittal sinus thrombosis where left orbital bruising had been noted. ${ }^{1}$ GTP has seen one other case of raised intracranial pressure in whom periorbital bruising was seen at presentation; that case also had sagittal sinus thrombosis. It remains possible that dural sinus thrombosis had occurred in case 2 but that this was no longer visible on imaging by the time she was scanned.

This new syndrome may be considered analogous to Terson syndrome where a branch of the central retinal vein ruptures into the vitreous following hyperacute rise in intracranial pressure after subarachnoid haemorrhage. ${ }^{2}$ Minor degrees of such haemorrhage (subhyaloid haemorrhages and minor vitreous haemorrhages) are seen occasionally in raised intracranial pressure from other causes but usually indicating a relatively abrupt onset ${ }^{3}$ (and GTP, personal observations). To our knowledge periorbital bruising has never been reported in cases of subarachnoid haemorrhage. It may be that intravitreal and subhyaloid haemorrhages in such cases occur due to a rise in the subarachnoid cerebrospinal fluid pressure causing in turn a rise in pressure in the ophthalmic vein. In cases of dural sinus thrombosis, however, there is likely to be a more generalised rise in intracranial venous pressure.

We believe this observation to be of importance because where papilloedema is seen in association with "black eyes" a cause of abrupt raised intracranial venous hypertension such as dural sinus thrombosis should be suspected. It is likely to be the case that such bruising is visible before papilloedema has developed. Furthermore, in case 1 it was assumed by a number of doctors that she had been assaulted, which led to inappropriate management.

\section{ACKNOWLEDGEMENTS}

We are grateful to both patients for granting us permission to publish the case reports and the photographs.

\section{Authors' affiliations}

S Hadjikoutis, C Carroll, G T Plant, Department of Neuro-

ophthalmology, Moorfields Eye Hospital, London, UK

The National Hospital for Neurology and Neurosurgery, London, UK

Competing interests: none declared

Correspondence to: Dr G T Plant, The National Hospital for Neurology and Neurosurgery, Queen Square, London, WCIN 3BG. UK; gordon@ plant.globalnet.co.uk

Received 3 April 2003

In revised form 26 October 2003

Accepted 8 November 2003

\section{REFERENCES}

1 Roy P-M, Gras E. Cerebral venous thrombosis. N Engl J Med 2003;349:1730.

2 Garfinkle AM, Danys IR, Nicolle DA, et al. Terson's syndrome: a reversible cause of blindness following subarachnoid haemorrhage. J Neurosurg 1992;76:766-71

3 McCasland B, Mendicino ME, Newman NJ. Subretinal haemorrhage in idiopathic intracranial hypertension. Br J Ophthalmol 1999;83:883-4. 